\title{
ESTUDIO ANALÍTICO NO LINEAL DE LOS MODELOS EXPLICATIVOS DE LA NUTRICIÓN VEGETAL Y SU VALOR PARA EL PROCESO DE ENSEÑANZA- APRENDIZAJE
}

\author{
Milagros Chávez Tortolero*
}

\begin{abstract}
This research paper consists of a non-sequential analytical study related to the history of the explanatory patterns of the vegetarian nutrition. The study is based on previous works by Thomas Kuhn and Gaston Bachelard who performed research on the continuity and discontinuity of the evolution of scientific knowledge. More specifically, the following explanatory models related to vegetarian nutrition will be categorized and analyzed. They are: the analogical plant-animal model (the humus and mineral nutritional sub models), the plant model acting as water transmutant, and the model where by plants react as "dephlogistical" agents to air. Consequently, this paper will also explain a number of fundamental ideas based on the value of the teaching-learning process and the understanding of these explanatory models as well as their evolution through time.
\end{abstract}

\section{RESUMEN}

Este trabajo consiste en un estudio analítico no lineal de la historia de los modelos explicativos de la nutrición vegetal. Se parte de un análisis sobre la continuidad y la discontinuidad de la evolución del conocimiento científico siguiendo los trabajos de Thomas Kuhn (1983) y de Gastan Bachelard (1967). Se categorizan y analizan los siguientes modelas explicativos: modelo analógico planta-animal (submodelos humus y nutrición minera)), modelo de las plantas como transmutadoras del agua y modelo de las plantas como deflogisticadoras del aire. Finalmente, se desarrollan algunas ideas fundamentales sobre el valor que tiene para el proceso de enseñanza-aprendizaje la comprensión de estos modelos explicativos y de su evolución histórica.

Palabras claves: Modelos explicativos de la nutrición vegetal, historia no lineal de las ciencias, enseñanza-aprendizaje de las ciencias y enseñanza-aprendizaje de la nutrición vegetal.

\section{INTRODUCCIÓN}

\footnotetext{
"Doctoranda de la Universidad de Québec en Montreal (Canadá); Licenciada en educación mención en Biología, Magíster en filosofía.
} 
El estudio de la manera como se han construido y como se siguen construyendo los conocimientos científicos puede aportar pistas fundamentales para, por un lado, transformar estos conocimientos en contenidos del proceso enseñanza-aprendizaje, y por otra parte, idear las mejores rutas para desarrollar este proceso.

El trabajo que a continuación se presenta es una revisión analítica de los modelos explicativos que a través de la historia han sido utilizados para explicar el proceso de la nutrición vegetal. Esta revisión tiene como objetivo fundamental abrir nuevas puertas para comprensión y mejoramiento del proceso de enseñanza-aprendizaje de este fenómeno natural.

En primer lugar, se han considerado las teorías de Thomas kuhn (1983) y de Gastan Bachelard (1967) como guías conductoras para elaborar una discusión relativa a la continuidad o discontinuidad de la historia de la ciencia. Seguidamente, se ha elaborado una categorización analítica de las explicaciones más importantes que se han producido históricamente sobre el fenómeno de la nutrición vegetal.

Finalmente se desarrollan algunas ideas fundamentales sobre el valar que tiene para el proceso de enseñanza-aprendizaje la comprensión de estos modelos explicativos y de su evolución histórica.

\section{El análisis de la historia de las ciencias: la acumulación continua o la evolución discontinua}

¿Cómo ha ocurrido la evolución de los conocimientos científicos? ¿Se trata acaso de un proceso de adición continua y homogénea de resultados de la ciencia? ¿O en cambio, se trata de una dinámica compleja caracterizada tanto por avances como por estancamientos y regresiones?

Si buscamos en nuestros recuerdos escolares, es muy posible que encontremos una idea de la historia como aquella disciplina que nos relataba la secuencia, más o menos exacta, de los hechos que ocurrieron en el pasado. En esta imagen, se hace manifiesta una confianza en la honestidad y en el punto de vista de aquellos que escriben la historia.

De acuerdo a lo anterior, la imagen que tenemos de la historia se asemeja a la de una película de larga duración donde los hechos se siguen los unos a los otros de una manera lineal y sumativa.

Según Kassou (1993), en la mayoría de los manuales escolares los contenidos históricos consisten en una sucesión de nombres de científicos, de fechas y de descubrimientos. Lo que se presenta en estos textos es una continuidad, sin ruptura ni debate, donde generalmente sólo se ponen en relieve las teorías triunfantes de cada época.

No obstante, si se hace una reflexión más profunda sobre la dinámica manifiesta en los hechos históricos, observamos que la historia nos es de ningún modo un proceso lineal y aditivo. Los hechos de la historia no se suceden los unos a los otros de una forma simple, sino que se interconectan y se relacionan a la manera de una red compleja de causas y efectos. La historia es un proceso dinámico donde todos los ámbitos del pensar humano se relacionan: la cultura, las tradiciones, la política, la religión, las ideologías, la ciencia, etc. 
Según Canguilhen (1983), la historia de las ciencias debe ser ante todo una teoría explicativa de la evolución de los hechos. Debe fundamentarse en el análisis crítico y profundo de estos hechos poniendo de relieve los saltos, los estancos, los errores y las regresiones de los postulados propuestos en cada momento.

Entre los autores más reconocidos por su intensa crítica a la historia lineal de la ciencia están Thomas Kuhn y Gaston Bachelard. Estos dos investigadores, así como otros estudiosos de la epistemología y de la historia de la ciencia, han puesto de relieve que la evolución de la ciencia no ha sido de ninguna manera un proceso lineal de adición continua de conocimientos. Por el contrario, estos autores coinciden en explicar que los caminos de la producción del saber científico han sido tortuosos y dinámicos (Kassou, 1993; Astolfi-Develay, 1989).

\section{Thomas Kuhn y las revoluciones científicas}

Según nos explica Kuhn en su libro La estructura de las revoluciones científicas (1983), existen dos modos bajo los cuales se desarrolla la investigación científica: la ciencia normal (que tiene su ámbito de trabajo en el cuadro del paradigma establecido y aceptado por la comunidad científica) y la ciencia extra-normal (que tiene lugar fuera de paradigma normal y que por lo tanto puede entenderse como marginal al consenso científico de la época). De este modo, lo que diferencia la ciencia normal de la ciencia extra-normal, es su adherencia o no a los paradigmas aceptados por la mayoría de los científicos de un momento histórico determinado.

Un paradigma es un sistema teórico-explicativo que sirve de piso a las investigaciones de una ciencia o de un conjunto de ciencias en un momento dado (kuhn, 1983). Como ejemplo de lo que es un paradigma podemos citar la teoría mecanicista del universo expuesta por Newton. Esta teoría se constituyó como la base sobre la que se fundamentaron todas las explicaciones de la física antes de la aparición de la teoría de la relatividad.

Según nos explica Kuhn, la ciencia es una actividad colectiva y no el fruto sumativo de los aportes hechos de manera individual y aisladamente por algunos científicos. Todo gran suceso de la ciencia tiene en su pasado un colectivo de investigadores que contribuyeron, de alguna manera, a la realización del mismo.

Este autor sostiene la idea de que la historia de la ciencia ocurre por saltos, es decir de manera discontinua. Estos saltos se deben a la aparición de teorías que se distinguen y/o se oponen a los paradigmas dominantes de la época. Es lo que Kuhn denomina revolución científica.

Kunh observó que la historia de la ciencia presentada en los manuales presenta regularmente sólo aquellos conceptos, teorías y experiencias que, por razones particulares y a veces ajenas a la misma ciencia, se impusieron sobre las otras posibilidades teóricas que existían. De esta manera, estos manuales dejan de lado todas las ideas "erróneas', las posiciones antagónicas y los debates que se presentaron paralelamente a las teorías triunfantes. Tal manera de presentar los hechos, nos impide analizar la verdadera dinámica de la ciencia y el sentido de sus revoluciones y sus saltos. 


\section{Gaston Bachelard: obstáculo epistemológico y ruptura epistemológica}

Gaston Bachelard en su libro La formation de l'esprit scientifique de 1967, expone su negación a la idea linear y acumulativa de la historia de la ciencia. Para él, la ciencia evoluciona de manera irregular, es decir, sufriendo momentos de estancamiento, de saltos y a veces de regresiones.

En su exposición, Bachelard pone al descubierto el valor fundamental del estudio de las hipótesis y representaciones "erróneas" que han formado parte del acontecer científico. A través de esta manera de estudiar la historia de las ciencias, Bachelard logró explicar la reiterativa resistencia a la transformación de ciertas construcciones del intelecto humano. Se9ún Bachelard, estas construcciones resistentes son aquellas que han sido elaboradas a partir de la experiencia primera, es decir de los contactos esenciales con la realidad inmediata.

En la evolución de la ciencia se han presentado muchas veces este tipo de ideas resistentes al cambio. Bachelard ha definido estas ideas como obstáculos epistemológicos de la ciencia. Como ejemplo se puede mencionar la teoría de los cuatro elementos de Aristóteles, la cual tuvo una fuerte persistencia entre los investigadores del siglo XVI y XVII.

El paso a niveles más avanzados de conocimiento sólo es posible si ocurre un desprendimiento, una indiferencia voluntaria, con respecto a estas construcciones primarias. Este acto genera lo que Bachelard identifica como una ruptura epistemológica. De esta manera, el motor de avance del conocimiento científico debe ser una permanente acción de sospecha y de ruptura con las ideas solidificadas y evidentes a los ojos.

En este sentido, la historia de la ciencia no puede ser lineal ni sumativa. Los obstáculos epistemológicos han frenado el avance del conocimiento por cierto tiempo, hasta que la duda y la sospecha sobre lo evidente, se hacen presentes y ocurre el proceso de ruptura epistemológica. Este último permite una transformación del conocer científico de manera que nuevas ideas e hipótesis pueden entrar en acción.

Bajo este ángulo de comprensión de la historia de las ciencias, a continuación se presenta un estudio de la evolución histórica de los modelos explicativos más cruciales de la nutrición vegetal.

\section{TIPOLOGÍA DE LOS MODELOS HISTÓRICOS DE LA NUTRICIÓN VEGETAL}

\section{El modelo general de la analogía planta-animal}

Hemos categorizado bajo el modelo de la analogía planta-animal dos submodelos. Estos se distinguen entre sí por sus explicaciones con respecto al procesamiento de sustancias. Ambos admiten que las plantas consumen sus alimentos de la tierra, pero la diferencia estriba en que para unos, estos alimentos están digeridos en la tierra misma, y para otros, es la planta la que se encarga de hacer estas transformaciones.

\section{El modelo del humus: el suelo como órgano digestivo}

Durante mucho tiempo la fisiología vegetal estuvo muy influenciada por la fisiología y la anatomía animal. A partir del conocimiento de algunos órganos y sus funciones en los 
animales, se buscaba explicar el funcionamiento de las plantas (Bryan Conant \& Nash, 1966; De Wit, 1993).

Aristóteles, en el siglo IV antes de Cristo, afirmaba que las plantas toman sus alimentos de la tierra a través de las raíces. En este modelo, la tierra es equiparada al estómago de los animales (Kassou, 1993). En el suelo ocurren todas las transformaciones digestivas, de tal manera, la planta incorpora por sus raíces los alimentos ya digeridos. Estos nutrientes ya transformados completamente en el suelo es lo que Aristóteles llamó el humus.

Muchos investigadores de distintas épocas, sobre todos aquellos que trabajaban en el área de la agricultura, se percataron de que las plantas crecían mejor en suelos ricos en materia orgánica descompuesta, es decir en suelos ricos en humus. Se suponía que aquella materia orgánica degradada por la tierra se convertía en sustancias alimenticias para las plantas. Estas por no tener estómago como los animales, debían incorporar tal cual estas sustancias y de este modo se explicaba su crecimiento (Bryan Conant \& Nash, 1966; De Wit, 1993).

El modelo explicativo del humus mantuvo su vigor durante toda la Edad media, así como durante el Renacimiento y parte de la Edad moderna. La razón de su éxito y permanencia se puede atribuir a dos razones fundamentales: en primer lugar, a la efectividad de estas explicaciones en el uso de abonos orgánicos en la producción agrícola, y en segundo lugar, al desconocimiento de la mezcla gaseosa del aire y su importancia en los procesos fisiológicos de la planta.

Sin embargo, con el paso del tiempo, el modelo del humus comenzó a ser insuficiente para dar explicación a ciertos fenómenos. Por ejemplo ¿cómo puede un árbol extraer sus alimentos de un mismo lugar durante tantos años? ¿Por qué algunas plantas pueden crecer instaladas sólo en el agua? Este tipo de preguntas se hicieron más numerosas cuando las ciencias químicas pusieron en el tapete la importancia de la conservación de las masas en los procesos químicos y fisiológicos. La cantidad de materia de la tierra no era suficiente para explicar la cantidad de materia desarrollada por un árbol.

\section{El modelo de las plantas capaces de digerir los nutrientes de la tierra: ¿dónde está el estómago de las plantas?}

La teoría del humus no fue la única que consideró la fisiología de las plantas como un equivalente de la fisiología animal. Luego de determinar que las propiedades de las sustancias extraídas por la planta del suelo (agua, sales, etc.) eran muy distintas a las que tenían los materiales localizados en la planta misma (madera, fibra vegetal, etc.), se llegó a la idea de que este grupo de seres vivos era también capaz de hacer una transformación de sustancias. Dicha transformación fue equiparada por algunos investigadores a la digestión animal.

A partir de esta idea, el problema sobre la nutrición de las plantas se centro en buscar en estos organismos las funciones y los órganos digestivos equivalentes a los encontrados en los animales.

De este modo, Malpighi (1628-1694), que era un biólogo mecanicista, planteó que los procesos de transformación de las sustancias de las plantas tenían lugar en las hojas, de manera análoga a como ocurre el proceso de digestión en el estómago de los animales. Según Malpighi, los materiales ya transformados en sustancias alimenticias en las hojas, 
viajan a través de la planta por medio de un sistema circulatorio equiparable al de los animales. De este modo, la savia es entonces vista como el líquido sanguíneo de las plantas (Bryan Conant \& Nash, 1966; De Wit, 1993).

Malpighi se percató también de que en las hojas de las plantas existían pequeños poros (estomas). Influenciado por el modelo de la fisiología animal, este investigador atribuyó a estos órganos una función excretora. Por medio de las estomas, las plantas desechan aquellos productos del proceso digestivo que no le son útiles a su desarrollo o al almacenamiento (Bryan Conant \& Nash, 1966; De Wit, 1993).

La idea de que la planta se alimenta fundamentalmente de la tierra se puede clasificar como un modelo derivado de lo que Bachelard denomina la experiencia primaria. Lo más inmediato a nuestros sentidos es que las plantas, que en su mayoría están fijadas al suelo, tienen órganos especializados para extraer sustancias. De este modo, la función principal de las raíces es la absorción de los nutrientes, transformados o no, del suelo. Las raíces representan así la boca de la planta en una analogía directa con la anatomía animal.

Esta representación puede ser explicada como un obstáculo epistemológico: para sobrepasarlo, es necesario tomar una distancia crítica frente a la evidencia inmediata dada a los sentidos. En el desarrollo histórico de la explicación del fenómeno de la nutrición vegetal, este distanciamiento se fue estructurando paulatinamente a partir de los diferentes caminos e intereses de investigación que iban emergiendo.

Las plantas son seres vivientes muy distantes a los animales. Los sistemas fisiológicos de ambos grupos no deben ser equiparados. Los procesos de nutrición, respiración, excreción, reproducción, etc, tienen caminos distintos en estos dos grupos de organismos. Esta consigna puede abrirnos a la creación de nuevas explicaciones. J. B. Van Helmont (1577-1644) fue uno de los que se atrevió a tomar esta distancia. A continuación explicamos cómo lo hizo y a que modelo explicativo llegó.

\section{El modelo de la transmutación y la nutrición de las plantas}

A finales de la Edad media e inicios del Renacimiento, el interés por dar explicación a los fenómenos naturales fuera del dogma religioso toma importancia. En esta acción se mezclan brujos y sabios. Los límites entre magia y ciencia no estaban todavía concebidos. De este modo nace la alquimia, una especie de corriente de ideas sobre la naturaleza que tenía carácter iniciático y místico. Los alquimistas creían en la posibilidad de transformar ciertos materiales innobles como el plomo, en otros considerados como nobles tal como el oro.

Esta transformación de los materiales era conocida con el nombre de transmutación. Para llevar a cabo este proyecto, los alquimistas realizaban largos trabajos experimentales donde utilizaban distintos compuestos que sometían a una variedad sin fin de procedimientos: cortar, rayar, pulverizar, calentar al fuego directo o indirecto, enfriar al frío nocturno etc. La química moderna tiene su origen en estas búsquedas y en estos procesos.

Uno de estos alquimistas, el belga J.B. Van Helmont (1577-1644) realizó una experiencia que está catalogada como fundamental para el avance del conocimiento sobre la nutrición de las plantas verdes (Bryan Conant \& Nash, 1966; De Wit, 1993). Como ya se dijo antes, la importancia de los trabajos de Van Helmont radica en su 
distanciamiento del modelo explicativo de la analogía planta-animal. Los trabajos de Van Helmont abrieron la posibilidad de que otros materiales, tales como el agua, fueran considerados como básicos para el desarrollo vegetal.

Van Helmont plantó una plántula de sauce de 5 libras en 200 libras de tierra que había secado antes de pesar para luego regarla con agua. Van Helmont dejó la planta a la luz y a la lluvia, sólo humedecía la tierra cuando era necesario. Al cabo de 5 años, el árbol pesaba 169 libras y 3 onzas. La tierra fue secada y pesada nuevamente. Su peso fue sólo dos onzas menor que al inicio de la experiencia. La conclusión de Van Helmont fue que la materia que el árbol había ganado en su crecimiento sólo podía provenir del agua, ya que la disminución de la cantidad de tierra era casi despreciable.

Basado en sus convicciones alquimistas, Van Helmont no tuvo problema en concluir que las plantas transforman (transmutan) el agua en madera, hojas y raíces. Este investigador consideraba que su experiencia con el sauce era la prueba suficiente de que la transmutación existía. De este modo, sus explicaciones sobre la nutrición de las plantas no fueron más adelante. Para Van Helmont y sus seguidores, el modelo de la transmutación se transformó en un obstáculo epistemológico que limitó el interés por profundizar en el estudio de la nutrición vegetal.

\section{El modelo del flogisto y los procesos de respiración y nutrición de las plantas}

Las investigaciones sobre la composición del aire y su importancia para la vida se desarrollaron a partir de experiencias hechas sobre los procesos de combustión. En el siglo XVII, un grupo importante de investigadores había puesto en boga la teoría según la cual los materiales combustibles contenían un principio de inflamabilidad que era liberado al aire durante el proceso de la combustión (Bryan Conant \& Nash, 1966; De Wit, 1993).

Este principio fue llamado flogisto (phlogiston) que en griego significa inflamable. Este principio no era considerado como una materia, sino que era más bien asimilado a una fuerza, a una especie de tendencia oculta, en este sentido, equiparable a la luz y al calor para ese momento. Cuando el aire está saturado de este principio se hace viciado y venenoso. Al estudiar la respiración animal, se llegó a la conclusión de que en ella también ocurría un enrarecimiento del aire. Se supuso entonces que la respiración era una combustión lenta en la que también era liberado el flogisto (Bryan Conant \& Nash, 1966; De Wit, 1993).

En 1771, Joseph Priestley (1733-1804), realiza una serie de experiencias a través de las cuales observa que las plantas tenían la capacidad de cambiar el aire viciado por la combustión y/o por la respiración animal. Priestley concluye entonces que las plantas tienen un tipo de respiración inversa a la de los animales ya que produce una deflogisticación del aire (Bryan Conant \& Nash, 1966; De Wit, 1993).

Las investigaciones de Priestley fueron seguidas muy de cerca por Antoine Laurent Lavoisier (1743-1794). Después de una multitud de experiencias rigurosamente controladas sobre la combustión y los gases involucrados, Lavoisier pudo aislar el oxígeno y describir algunas de sus características más generales. Aún sin poder determinar la naturaleza de los gases carbónicos, este investigador estimó que el aire era una mezcla de dos tipos de gases, el oxígeno o gas respirable, y el azote (del griego a = sin, y zooè = vida) o gas tóxico. 
La rigurosidad de Lavoisier en cuanto a la medida y control de las masas de los materiales antes y después de cada combustión y de cada reacción, le hicieron darse cuenta de que después de la combustión la masa del material combustible era mayor que al comienzo de la experiencia. Tal situación le indujo a pensar que durante la combustión, en vez de salir algo (flogisto) del combustible, hay una masa que se suma al mismo para formar la ceniza (oxígeno).

Este hecho permitió a Lavoisier considerar que tenía los argumentos suficientes para intentar destronar el modelo del flogisto. Sin embargo, este modelo de explicación continuó siendo la base de muchas investigaciones importantes sobre la nutrición de las plantas. De este modo, J. Ingenhousz (1 730-1 799) explicó que las plantas sólo producen aire deflogisticado en sus partes verdes y en presencia de luz. Del mismo modo que J. Senebier (1 767-1 845) exponía que las plantas producen aire deflogisticado sólo en la presencia de flogisto, de "aire viciado" (kassou, 1993).

El abandono del modelo del flogisto sólo fue posible mucho tiempo después, cuando los avances en el nuevo modelo de la química propuesta por Lavoisier habían alcanzado suficiente coherencia explicativa. Es así como el modelo del flogisto da paso al modelo de la oxido-reducción (Bryan Conant \& Nash, 1966; De Wit, 1993).

Los trabajos sobre el aislamiento y la determinación de las propiedades del gas carbónico permitieron avanzar los estudios sobre la importancia de este gas en el proceso de la nutrición vegetal. En 1782, Senebier (1742-1809) realiza un conjunto de experiencias donde se percata que en la ausencia de este compuesto las plantas no producen oxígeno (Kassou, 1993).

Sé comienza entonces a manejar la idea de que las plantas utilizan el gas carbónico en un proceso de síntesis para construir compuestos más complejos. Se hace evidente también que este proceso tiene lugar sólo si la planta está en presencia de luz. A partir de este momento, comienza a desarrollarse el modelo actual de fotosíntesis bajo los criterios de la ciencia normal según los define Thomas kuhn.

\section{El valor didáctico del análisis de los modelos explicativos de la nutrición vegetal}

Muchos son los investigadores de la didáctica de las ciencias que han puesto de relieve la importancia de la historia de las ciencias en los procesos de enseñanzaaprendizaje de los contenidos científicos (Astolfi-Develay, 1989; estudiantes; Gil PérezGuzmán Ozamis, 1993; Kassou-Souchon, 1992). La idea central que se maneja a este respecto gira en torno a la relación que se estipula existe entre la evolución histórica del conocimiento científico y el desarrollo cognitivo de las personas. Se trata, en cierta medida, del ya conocido proyecto piagetiano: el establecimiento de una relación entre la ontogénesis del conocimiento y la evolución histórica del mismo (filogénesis) (Chávez, 1998).

El comprender la dinámica de la evolución histórica de los modelos explicativos nos permite ubicar los nudos fundamentales de la transformación de los mismos. Siguiendo los postulados de Bachelard (1967), se trata de comprender cuales fueron los obstáculos epistemológicos de las explicaciones y las rupturas que fueron necesarias para su superación. 
El trabajo de la planificación didáctica consiste entonces en hacer el puente entre estos nudos fundamentales de la historia de la ciencia y el desarrollo cognitivo de los estudiantes. En otras palabras, se trata de diseñar dinámicas del proceso de enseñanza aprendizaje a partir de ciertas rupturas epistemológicas de la historia de la ciencia.

Los modelos históricos identificados en este trabajo: la analogía planta-animal, la teoría del humus, la nutrición mineral, las plantas y la transmutación, y la teoría del flogisto, representan un avance en esta tarea didáctica. Ellos pueden servir de base al diseño de estrategias para la enseñanza-aprendizaje de la fotosíntesis y de la respiración.

Algunas investigaciones (Wandersee, 1985; Baker-Carr, 1992; Cañal, 1990) han revelado que los modelos explicativos sobre la nutrición vegetal de los estudiantes (niños, jóvenes y adultos) se asemejan a los modelos explicativos que han sido tipificados en este trabajo. Por ejemplo, Wandersee (1985) realizó un estudio sobre las concepciones de un grupo de adolescentes sobre la fotosíntesis. La idea de que las plantas se alimentan del suelo fue una de las concepciones de mayor frecuencia y resistencia. La explicación de este hecho puede atribuirse a que, en la vida cotidiana, se observa que la gran mayoría de los vegetales tienen órganos especializados en extraer sustancias del substrato donde se localizan. Se infiere entonces por analogía con los animales que las raíces son la boca de las plantas y la tierra su alimento.

El proceso de transformación de este modelo en la historia puede servirnos de guía para idear estrategias que genere en los estudiantes una ruptura epistemológica frente a lo evidente en este fenómeno. De este modo, Campestrini (1992) y Sauvageot-Skibini (1997) han propuesto estrategias didácticas en las cuales se analiza el experimento del sauce de Van Helmont. Los resultados de estos trabajos muestran que en efecto, a partir deL análisis de este experimento, se genera en los estudiantes una crisis conceptual (ruptura epistemológica) con respecto al modelo inicial espontáneo de la analogía plantaanimal.

Es posible que seguidamente a esta ruptura un nuevo modelo se instale. En éste, el agua es un recurso suficiente al crecimiento de la planta. Es conveniente entonces tomar pistas de la dinámica histórica de la explicación del fenómeno. Los estudiantes pueden ser conducidos a una reflexión sobre los materiales y sus características. Por qué el agua no puede transformarse en madera, por ejemplo.

De manera paulatina se induce en los estudiantes un proceso ascendente de abstracción. Se trata de producir en ellos un distanciamiento progresivo con respecto a la realidad inmediata y al sentido común cotidiano, para de este modo abrirlos a la comprensión de que la planta utiliza un gas invisible, el $\mathrm{CO}^{2}$, para construir (sintetizar) sus propias sustancias alimenticias, utilizando como recurso energético la luz.

Se observa de esta manera que en el proceso didáctico sobre la nutrición vegetal se necesita trabajar con los estudiantes no solamente las explicaciones de este fenómeno sino también de otros conceptos, modelos y fenómenos, tales como el de materia (tipos, estados, masa, volumen, etc.), energía, reacción química, espectro luminoso, etc. Lo que se sugiere, es que se inicie a los niños, desde muy temprana edad, a la búsqueda de explicaciones a los diferentes fenómenos de la naturaleza vinculados a la nutrición de las plantas. Luego, en los niveles de secundaria, esta sensibilización será útil para la comprensión de los elementos más abstractos del modelo de la fotosíntesis. 
La iniciación temprana a la explicación de los fenómenos vinculados con la nutrición vegetal es posible a través del diseño de estrategias didácticas, desde el preescolar y la escuela primaria, que favorezcan la discusión, el análisis, los conflictos conceptuales, la imaginación, la duda metodológica y la experimentación. Estas estrategias deben partir de la experiencia cotidiana y de los modelos de los alumnos.

Esta iniciación temprana abre la puerta a un proceso de abstracción paulatino que debe acentuarse en la adolescencia. En efecto, ya en la escuela secundaria se debe insistir con más ahínco en la necesidad de tomar una distancia crítica frente a lo evidente para darle explicación a los fenómenos.

A través de la elaboración y discusión de experiencias y estudios que muestran la necesidad de luz de las plantas, el intercambio de gases de éstas y el medio y los cultivos hidropónicos, es posible conducir los adolescentes a una ruptura epistemológica con respecto a los modelos explicativos espontáneos.

Sin embargo, es importante señalar que estos modelos espontáneos, como por ejemplo el de la analogía planta-animal, son altamente resistentes. Ello se explica por su gran utilidad en las explicaciones de una multitud de fenómenos cotidianos, tales como el uso de abonos orgánicos en la agricultura y en la formación de suelos en los bosques.

No obstante, en la actualidad, estamos también en presencia de fenómenos sumamente complejos cuya comprensión por parte de la opinión pública cobra un valor sin precedente ya que esto garantiza la participación en los debates y las acciones comunitarias. Tal es el caso de la capa de $\mathrm{CO}^{2}$, el balance de $\mathrm{CO}^{2}$ del planeta, la deforestación y el uso desmedido de combustibles fósiles, el efecto invernadero y el recalentamiento de la tierra Estos fenómenos están en íntima relación con el proceso de nutrición vegetal, pero este hecho no es evidente si se continúa aferrado a un modelo analógico de la planta-animal. Es ésta una de las razones fundamentales que debe incitarnos a conducir a los estudiantes a una torna de distancia con respecto a estos modelos espontáneos.

\section{Conclusión}

Este trabajo ha permitido realizar un estudio analítico de los modelos explicativos que a través de la historia han servido para darle sentido al proceso de nutrición de las plantas.

Se ha partido de un análisis sobre la continuidad y la discontinuidad de la evolución del conocimiento científico. Siguiendo los trabajos de Thomas Kuhn y de Gaston Bachelard, se ha puesto de relieve el sentido dinámico de la historia de las ciencias. La presencia reiterada de obstáculos y rupturas epistemológicas en la evolución del conocimiento científico, así corno de las revoluciones científicas, nos permite inducir el carácter de discontinuidad de la misma.

Para afinar este estudio sobre la evolución de las explicaciones hechas sobre el fenómeno de la nutrición vegetal, hemos utilizado la técnica de categorizar estas explicaciones como modelos.

Se observó que ciertos modelos lograron permanecer en el tiempo presentando variantes pero conservando siempre su esencia explicativa. Tal es el caso del modelo analógico planta-animal. El modelo del humus fue reemplazado progresivamente por el de 
la nutrición mineral, pero en esencia, se seguía conservando la idea de que las plantas toman su alimento de algo exterior a ellas y palpable a nuestros sentidos, la tierra.

Este modelo tuvo la oportunidad de entrar en una crisis a partir de las experiencias del sauce de Van Helmont. Sin embargo, el modelo de la transmutación se convirtió en un nuevo obstáculo epistemológico.

Fue necesario que aparecieran los resultados de investigaciones realizadas en dominios distintos al de la fisiología vegetal. Los trabajos sobre la combustión permitieron agregar nuevas luces sobre la comprensión de este fenómeno. Luego, con la aparición de la química de Lavoisier, la apertura al modelo de síntesis de los materiales como proceso de la nutrición vegetal y la importancia de los gases del aire en este proceso comenzaron a abrirse paso.

Este análisis ha permitido mirar la didáctica de la fotosíntesis bajo perspectivas tales como la multidisciplinaridad que este modelo explicativo expone y la secuencia en grados de abstracción que se hace necesaria. Esto nos lleva a su vez a reflexionar sobre los factores de maduración de los estudiantes y sobre las estrategias didácticas que se pueden aplicar a respecto.

\section{BIBLIOGRAFIA}

Astolfi J. P. y Develay, M. (1989). La didactique des sciences. Paris: Presses Universitaires de France.

Bachelard, G. (1967). La formation de l'esprit scientifique. Paris: Librairie philosophique Vrin.

Baker, M. y Carr, M. (1992). Un modèle d'enseignement génératif de la photosynthèse. Aster (15), pp. 33-54.

Brian Conant, J. y Nash, L. (1966). Harvard Case Histories in Experimental Science. Cambridge, Massachusetts: Harvard University Press.

Campestrini, P. (1992). Sortir de la logique de Van Helmont. Aster (15), pp. 7-31.

Canguilhen, G. (1983). Études d'histoire et de philosophie des sciences. Paris: Librairie Philosophique Vrin.

Cañal, P. (1990). La enseñanza en el campo conceptual de la nutrición de las plantas verdes: un estudio didáctico en la educación básica. Tesis de doctorado, Sevilla:Université de Sevilla.

Chávez, M. (1998). En torno al origen de la representación del espacio en la epistemología genética de Jean Píaget. Tesis de Maestría, Mérida- Venezuela: Universidad de los Andes.

De Wit, H. (1993). Histoire du développement de la biologie. Vol II. Lausanne: Presses polytechniques et universitaires romandes. 
Gil Pérez, D. y De Guzmán Ozamis, M. (1993). La enseñanza de las ciencias y las matemáticas. Madrid: Popular.

Ingen-Housz, J. (1779). Experiments Upen Vegetables. London.

Kassou, S. et Souchon, C. (1992). Utilisation des aspects historiques dans l'enseignement de la photosynthèse. Aster (15), pp. 55-73.

Kassou, S. (1993). Eléments pour l'analyse didactique du statut de l'expérience dans l'enseignement de la biologie: le cas de la photosynthèse. Thèse de doctorat. Paris: Université de Paris VII.

Kuhn, T. (1983). I.a structure des révolutions scientifiques. Trad. Par Laure Meyer. Paris: Champs Flammarion.

Lavoisier, A. L. (1770). “Sur la nature de l'eau'. In Lavoisier's Euvres, vol. 2. Paris.

Malpighi, M. (1675). “Anatomie Plantarum’. In Opera Omnia. London.

Priestley, J. B. (1772). Observation en Different Kinds of Air. Vol. 1. $3^{\mathrm{a}}$ ed. London, 1881.

Sauvageot-Skibini, M. (1997). Ce que le professeur prévoit,... ce qui se passe réellement. Aster, (25), pp. 93-112.

Senebier, J. (1782). Mémoires physicochymiques. Genéve.

Van Helmont, J.V. (1648). Ortus Medicinae. (Leyden, 1648). Traducción al inglés por J. Chandler (1662), London: Oriatrike.

Wandersee, J. (1985). Can the Histo,y of Science Help Science Educators Anticipate Students' Misconceptions. Journal of Research in Science Teaching, 23 (7), pp. 581597. 